\title{
Obtención, separación y purificación de almidón a partir de Colocasia esculenta para su uso como excipiente en formas farmacéuticas
}

\section{Obtaining, separating and purifying starch from Colocasia esculenta for use as an excipient in pharmaceutical forms}

\author{
Leobardo Reyes-Velasco, ${ }^{1 *}$ Érick Pacheco-Rodríguez ${ }^{1}$, \\ Asela Garnica-Sánchez ${ }^{1}$, José Ángel Cueva-Villanueva ${ }^{1}$ \\ y Arturo Zapién-Martínez ${ }^{1}$ \\ Fecha de recepción: 14 de diciembre de 2018 \\ Fecha de aceptación: 5 de marzo de 2019
}

\begin{abstract}
Resumen - La malanga (Colocasia esculenta) es un producto de grado alimenticio que tiene una cantidad considerable de almidones y que ofrece una gran versatilidad de aplicaciones a bajo costo. Puede ser utilizada en diversos tipos de industrias y para un sinnúmero de propósitos. En este trabajo se utilizó el almidón extraído de C. esculenta con el objetivo de evaluar su capacidad aglutinante como una alternativa para elaborar formas farmacéuticas sólidas (tabletas) en comparación con el almidón de papa. A los almidones extraídos de malanga y papa se les realizaron ensayos de identidad: porcentaje de humedad, pH y tamaño de partícula, así como pruebas reológicas: velocidad de flujo, ángulo de reposo, densidad aparente, densidad compactada e índice de Hausner. Se elaboraron tabletas con ambos almidones, a las cuales se les midió dureza, peso, diámetro, espesor y tiempo de desintegración. Para establecer las diferencias entre los grupos de tabletas se realizó un ANOVA y prueba post hoc de Tukey. Se concluye que el almidón extraído de la harina de $C$. esculenta puede usarse como excipiente en la elaboración de tabletas o comprimidos.
\end{abstract}

Palabras clave:

Malanga, Colocasia esculenta, almidón, tabletas.
Abstract - Taro (Colocasia esculenta) is a food grade product that has a considerable amount of starch, which offers great versatility of applications at low cost. It can be used in different types of industries and has many applications. In this paper the starch extracted from $C$. esculenta was used with the purpose of evaluating its binding capacity as an alternative to be used in the preparation of solid pharmaceutical forms (tablets) in comparison with potato starch. The starches extracted from taro and potato were tested for identity: percentage of humidity, $\mathrm{pH}$ and particle size; as well as rheological tests: flow velocity, resting angle, bulk density, compacted density and Hausner index. Tablets with taro starch and potatoes were made, to measure its hardness, weight, diameter, thickness and disintegration time. To establish the differences between the groups of tablets (taro, potato and commercial acetylsalicylic acid -Aspirinas control), an ANOVA and Tukey post hoc test were performed. It is concluded that the starch extracted from the $C$. esculenta flour can be used as an excipient in the manufacture of tablets.

\section{Keywords:}

Taro, Colocasia esculenta, starch, tablets.

${ }^{1}$ Facultad de Ciencias Químicas, Universidad Autónoma "Benito Juárez" de Oaxaca (UABJO). Av. Universidad s/n, col. Cinco Señores, C.P. 68120, Oaxaca de Juárez, Oax., México. *Correo electrónico: Ireyes.cat@uabjo.mx 


\section{Introducción}

a malanga (Colocasia esculenta) es una planta perenne, hidrófila, su sistema radicular es ávido de agua y resistente a la asfixia; en ocasiones permite su cultivo en zonas pantanosas o en alternancia con el arroz en las mismas condiciones de nivel de agua. Tiene un antecedente muy antiguo, su historia puede seguirse hasta las culturas neolíticas más primitivas. Se expandió en el viejo mundo, cuya domesticación pudo hacerse en India-Indochina, en donde aún se encuentran poblaciones silvestres; la siembra se llevó hacia el este hasta los confines de Polinesia, Hawái y Nueva Zelanda, y en otra dirección hasta Filipinas, China y Japón (Musumara, Scarlala y Cirma, 1985).

En México se cultiva en los estados de Oaxaca, Veracruz, Tabasco y Yucatán, con una producción de 40 ton/ha; la producción anual en países que la cosechan es hasta de 84 ton/ha (FAO, 2014). Con respecto al uso habitual de la malanga, la Organización para la Alimentación y la Agricultura (FAO) y la Organización Mundial de la Salud (OMS), organismos especializados dependientes de las Naciones Unidas, relacionan al almidón con la dieta, la nutrición y la prevención de enfermedades crónicas, incluso se han hecho algunas recomendaciones para el consumo de carbohidratos (FAO y WHO, 2002). El almidón es un componente importante de los alimentos vegetales, que además es materia prima significativa para la industria. En Malawi es ampliamente utilizado para fabricar diversos productos, tales como alimentos, textiles, artículos farmacéuticos, células secas y adhesivos. El empleo del almidón depende de sus propiedades funcionales y fisicoquímicas (Peroni, Rocha y Franco, 2006; Srichuwong, Sunarti, Mishima, Isono y Hisamatsu, 2005; Riley, Wheatley y Asemota, 2006; Tester, Yusuph, Millam, Davies, 2004):

a) En la industria alimentaria es comúnmente usado como agente espesante, aglutinante, estabilizante, gelificador, secante y carga. Se encuentra en aderezos, mayonesas, alimentos preparados, rellenos de pastel, harinas preparadas, botanas, budines, alimentos infantiles, atoles, entre otros.

b) En la industria del papel y cartón, para proporcionar resistencia interna al papel y como adhesivo en la fabricación de empaques de cartón corrugado de todo tipo.

c) En la industria cervecera se emplea como fuente de carbono para procesos de fermentación, por su elevado extracto fermentable de más de $102 \%$ en biomasa seca (b.s.).

d) En la industria farmacéutica funciona como agente lubricante, aglutinante, diluyente, desintegrante y/o excipiente en la elaboración de tabletas o comprimidos (Langeland y Burks, 1998).

En México, su cultivo ha aumentado pero su uso ha sido limitado debido a su corta vida poscosecha (Instituto Nacional de Nutrición, 1999; Agbor-Egbe y Rickard, 1991). La elaboración de harinas compuestas a través de la mezcla de harina de malanga con otras representa una alternativa de uso. En general, las harinas de cereales, leguminosas, tubérculos o frutos secos pueden utilizarse en la industria de alimentos como ingrediente en botanas, salsas, cremas y pastas, entre otros. Sin embargo, la aplicación de estas harinas se relaciona directamente con su composición y propiedades funcionales, que dependen de la fuente y condiciones del cultivo (Dendy, 2001).

Es amplia el área de estudio en cuanto a las características que hacen más conveniente la distribución de estos productos y más fácil su transformación, que ponen de relieve las propiedades físicas, químicas y orgánicas requeridas por los mercados a los que se destinan. Para los almidones que carezcan de las peculiaridades funcionales innatas buscadas, es necesario impulsar e implementar técnicas que permitan agregarles valor o modificarlos para que puedan competir internacionalmente (Gujska, D-Reinhard y Khann, 1994; Connors, Amidon y Stella, 1986).

Son muy bien conocidas las propiedades aglutinantes, desintegrantes y diluyentes de los 
almidones; sin embargo, en su estado normal carecen de la compresibilidad y fluidez necesarias para emplearse en compresión directa. Los almidones para la industria farmacéutica consisten en una mezcla de gránulos de almidón intactos y gránulos parcialmente hidrolizados, subsecuentemente aglomerados en estado húmedo, resultando un material con un contenido de humedad extremadamente alto (12 a 13\%), que sin embargo no parece acelerar la descomposición de los fármacos hidrolábiles. Es un excipiente con aceptables propiedades aglutinantes, que sufre deformación plástica y cohesiona por fuerzas intermoleculares. No obstante, debido a una significativa deformación elástica precisa de elevadas fuerzas de compresión para formar tabletas duras; produce compactos con buena capacidad desintegrante, sin menoscabo de fluidez ni de compresibilidad en las formulaciones de compresión directa. Generalmente se usa como desintegrante-diluyente antes que como aglutinantediluyente (Arancibia, Chávez, Costa, Nella y Thielemann, 1994).

Los almidones en estado seco constituyen los diluyentes insolubles más utilizados, por su bajo precio y por sus propiedades desintegrantes, adsorbentes y aglutinantes. Su mayor inconveniente es que adsorben fácilmente humedad atmosférica, debido a que presentan valores de humedad de equilibrio relativamente altos (11-18\%), pudiendo causar problemas de inestabilidad en fármacos hidrolábiles. Actualmente se recurre al almidón de maíz y a ciertos derivados modificados por tratamientos físicos o químicos para mejorar algunas de sus características, como la compresibilidad, la capacidad de disgregación y el flujo (Sta-Rx $1500^{\circledR}$, Cellutab ${ }^{\circledR}$, Primoje( $\left.{ }^{\circledR}\right)$, y que tienen aplicación en la compresión directa (Vila Jato, 1997).

Por lo anterior, el objetivo del presente trabajo fue evaluar la capacidad aglutinante del almidón extraído de la harina de malanga (Colocasia esculenta), como una alternativa para ser empleada en la elaboración de formas farmacéuticas sólidas (tabletas).

\section{Materiales y métodos}

La materia prima se colectó directamente en campos de cultivo de Tuxtepec, Oaxaca, México. Se eligieron matas salteadas a una distancia considerable entre una y otra. Una vez obtenidos los cormos de malanga se les practicó una revisión física interna y externa para seleccionar los más sanos y grandes; posteriormente se les lavó el exterior en forma manual, en platones y con la ayuda de un cepillo de cerdas blandas; se retiró la cáscara manualmente con un cuchillo. Una vez peladas, se lavaron nuevamente con agua limpia y se procedió a rallar con un rallador de plástico. Una vez rallada toda la materia prima se sumergió en una solución de bisulfito de sodio (NaHSO3), a una concentración de 100 a 200 ppm por un tiempo de 20 minutos. Luego se deshidrató y secó totalmente mediante una estufa de secado. La materia seca se premolió con una licuadora y se molió finamente con un molino que constaba de cono, embudo, placa de vidrio, recolector y aplicador.

Para obtener almidón de papa se colocó una porción de los tubérculos pelados, limpios y trozados con una cantidad igual de agua en una licuadora, mezclándose a velocidad media por un minuto. El homogeneizado se filtró y el residuo se resuspendió en dos volúmenes de agua destilada, repitiéndose el procedimiento hasta obtener un filtrado claro, indicando que todo el almidón había sido extraído. Los filtrados se centrifugaron a $447.2 \mathrm{~g}$ durante 15 minutos, descartándose el sobrenadante. El residuo se lavó varias veces con $10 \mathrm{~mL}$ de agua destilada para eliminar otros constituyentes presentes. El almidón extraído se secó a $45^{\circ} \mathrm{C}$ por 24 horas, posteriormente se tamizó con malla $60(250 \mathrm{~mm})$ y se almacenó en frascos de plástico con cierre de tapa hermética para su posterior uso (Rincón, Araujo, Padilla y Martín, 2000). A los almidones extraídos de la malanga y la papa se les practicaron los ensayos de identidad (Secretaría de Salud, 2000): porcentaje de humedad (Ansel y Popovich, 1993), pH (Braverman, 1992) y tamaño de partícula, así como las pruebas reológicas: velocidad de flujo, ángulo de reposo, densidad aparente, densidad 
compactada e índice de Hausner (Secretaría de Salud, 2000; Parrot, 1971; Ansel y Popovich, 1993). Las variables se midieron en una sola ocasión.

Para elaborar las tabletas se utilizó una prensa hidráulica de fabricación propia, con un punzón plano de 12.9 mm, durante 10 segundos (Garzón, 2006). También se hicieron pruebas para determinar las fuerzas de compresión que se aplicaron en el experimento. A cada comprimido fabricado se les hizo pruebas de tiempo de desintegración, espesor, diámetro, dureza, y peso. Cada una se realizó por duplicado, a excepción de la prueba de tiempo de desintegración, la cual fue por triplicado.

Con la finalidad de determinar las diferencias entre los grupos de tabletas (malanga y papa), se llevó a cabo un ANOVA y prueba post hoc de Tukey; como grupo control se utilizaron tabletas de ácido acetilsalicílico de la marca comercial Aspirina de Bayer.

\section{Resultados}

A través de los ensayos de identidad se obtuvo una jalea blanquecina y translúcida, que se coloreó mediante una solución reactiva de yodo a un tono azul violeta que viró a azul intenso, indicando la presencia de almidón. En relación con la determinación del porcentaje de humedad, pH y tamaño de la partícula, ambos almidones presentaron valores similares, lo que indica homogeneidad entre ambas especies vegetales. Con respecto a las pruebas reológicas, los dos cumplen con las especificaciones para dichas pruebas y fueron aptos para su uso en la elaboración de tabletas (cuadro 1). Las tabletas se fabricaron por compresión directa, a una presión de 2.5 toneladas para las de almidón de papa y de 3.5 toneladas para las de almidón de malanga, debido a que este último soporta más la fuerza de compresión y el de papa a ese grado de fuerza resulta en tabletas demasiado frágiles.

En cuanto al análisis de las tabletas, se observó que el tiempo de desintegración y peso de las elaboradas con almidón de malanga fueron estadísticamente menores en comparación con las de papa y las de aspirina. Acerca del espesor y la dureza, se observó que las tabletas de malanga presentaron valores superiores estadísticamente significativos con respecto a los otros grupos. Con relación al diámetro, no se observaron diferencias importantes (cuadro 2).

\section{Discusión}

La malanga (C. esculenta), al igual que otros tubérculos, constituye un alimento esencialmente energético debido a su contenido de almidón, fibra dietaria, vitamina B6 y manganeso. Los tubérculos y los rizomas, incluyendo varios tipos de papa, yuca y malanga, son relativamente bajos en proteína (hasta 2\%), pero aportan gran variedad de vitaminas y minerales (hierro, fósforo, sodio y calcio) (Onwueme, 1999).

La malanga se cultiva en zonas tropicales, sus cormos contienen cantidades considerables de almidón (70-80 g/100 g de malanga seca) (Tattiyakul, Asavasaksakul y Pradipasena, 2006). Se obtuvo una jalea blanquecina y translúcida acorde con lo reportado en la literatura (Rodríguez-Miranda et al., 2011); se comprobó la presencia de almidón, de acuerdo con los ensayos de identidad descritos en los métodos generales de análisis de la Farmacopea de los Estados Unidos Mexicanos (Secretaría de Salud, 2000), dando un resultado positivo.

Los valores obtenidos en la determinación del porcentaje de humedad para los almidones de papa y malanga fueron similares, lo que muestra la uniformidad de este parámetro. Una de las causas a las que se puede atribuir la diferencia mínima encontrada es debido a la variación en el contenido de carbohidratos, principalmente almidón, ya que se ha encontrado que a mayor contenido de estos compuestos puede haber una mayor cantidad de agua absorbida (Tjahjadi, Lin y Breene, 1988). El valor de $\mathrm{pH}$ obtenido coincide con otros reportes. Al respecto, Falade y Okafor (2013) calcularon los valores de $\mathrm{pH}$ en cinco variedades de almidón provenientes de C. esculenta cultivada, cuyos rangos oscilan entre $4.76 \pm 0.01$ a $5.30 \pm 0.01$ y valores de $\mathrm{pH}$ de $5.27 \pm 0.02-6.78 \pm 0.00$ reportados 
por Rodríguez-Miranda, Ruiz-López, Herman-Lara, et al. (2011), mientras que el valor de pH de almidón de C. esculenta registrado por Mweta, Labuschagne, Koen, Benesi y Saka (2008) fue de 6.3. Determinar el pH es importante para conocer el factor ácido, que es un indicador de la velocidad de conversión del almidón a dextrina (Holleman y Aten, 1956).

En lo referente al ángulo de reposo, se han establecido algunos límites para esta propiedad, aunque no siempre resultan óptimos, ya que depende mucho del tipo de material (Linoya, Gotoh y Higashitani, 1995; Carteasen, 1990). De acuerdo con los valores del ángulo de reposo del almidón de papa, se considera como un polvo móvil, mientras que el de malanga califica como poco móvil (cuadro 3); esta medición es relativa de la fricción entre partículas del polvo, pero también se puede medir la cohesividad de finas partículas, la cual también se ve afectada por el tamaño de partícula (Linoya, Gotoh y Higashitani, 1995).

El almidón de papa tiene un valor ligeramente mayor en la velocidad de flujo con respecto del almidón de malanga, esto indica que ofrece una fluidez ligeramente más rápida que el de malanga. Sin embargo, se debe considerar que depende de las propiedades del polvo, tales como superficie, distribución de tamaño de partícula, forma y porosidad, entre otras. Los polvos en compresión deben mostrar una fluidez que asegure un llenado preciso y rápido de la matriz (Parrot, 1971).

La densidad aparente es una medida de la pesadez de las muestras sólidas. Es importante para definir los requerimientos de empaque, manipulación de los materiales y aplicación en la industria alimentaria. La densidad aparente depende del tamaño de partícula de las muestras; en este estudio, la del almidón de papa fue relativamente mayor a la del de malanga, situándose dentro del rango reportado por Falade y Okafor (2013), cuyos valores van desde $0.14 \mathrm{~g} /$ $\mathrm{mL}$ hasta $1.18 \mathrm{~g} / \mathrm{m}$ y más próximo al valor reportado por Kaushal, Kumar y Sharma (2012) de 0.69 g/ mL; mientras que Kaur, Kaushal y Sandhu (2013) hallaron valores $0.69 \pm 0.03 \mathrm{~g} / \mathrm{mL}$ y $0.99 \pm 0.02 \mathrm{~g} / \mathrm{mL}$ para la densidad aparente del almidón de malanga y de papa, respectivamente. Empero, esta diferencia no afecta la aceptación del almidón de malanga como buen excipiente, ya que tal propiedad sólo permite determinar el volumen ocupado por el material y elegir el tamaño del equipo a utilizar para el mezclado.

Respecto de los valores obtenidos para la densidad compactada, al evaluar este parámetro se pudo deducir que el almidón de malanga tiene mayor compresibilidad que el de papa, ya que la compactibilidad aumenta a medida que el valor incrementa (Parrot, 1971). El índice de Hausner es la relación de la densidad compactada entre la densidad aparente, es muy usado en la caracterización del polvo; se trata de una medición de fricción interparticular, donde los valores próximos a 1 indican buena fluidez y altos valores indican elevada cohesión interparticular, lo cual reduce las propiedades de flujo (Parrot, 1971; Ansel y Popovich, 1993). Los almidones de papa y malanga tienen buena fluidez, ya que sus valores están cercanos a 1 .

El tamaño de los gránulos de almidón en el proceso de cultivos alimentarios es importante para el alimento, ya que afecta su comportamiento durante el procesado. Por ejemplo, los pequeños son más resistentes a la ruptura y pérdida de orden molecular (Dreher, Dreher y Berry, 1984). Se determinó el tamaño de partícula del almidón de malanga y de papa midiendo el diámetro medio de las partículas a través del análisis granulométrico por tamización. El tamizado es el método generalmente empleado para conocer la granulometría de los polvos de uso farmacéutico, que es particularmente útil cuando la mayoría de las partículas son mayores de $100 \mu \mathrm{m}$, por lo que el tamaño encontrado para ambos almidones es mayor a lo reportado en la literatura: 6.47-13.63 $\mu \mathrm{m}$ con microscopía electrónica (Falade y Okafor, 2013). Pérez, Schultz y Pacheco (2005) reportan que los gránulos de almidón de Colocasia esculenta son de forma redondeada, elipsoidal truncada y poliédrica de 0.5 a 5.0 um; Nwokocha, Aviara, Senan y Williams (2009) 
reportaron que miden $6.54 \pm 2.23 \mu \mathrm{m}$. Sin embargo, este tamaño de partícula es adecuado para la utilización de almidón como aditivo en la elaboración de tabletas.

En cuanto al tiempo de desintegración de las tabletas, se encontraron valores distintos para cada tipo, lo que indica una diferencia estadística significativa entre las tres $(P \leq 0.05)$ y las que más rápido se desintegran son las elaboradas con almidón de malanga. El efecto casi nulo que mostró la fuerza de compactación en la desintegración de las tabletas revela que la porosidad del compacto y la fuerza de unión entre las partículas no son los factores determinantes; aparentemente el proceso de desintegración está controlado por el espesor y cohesividad de la capa de gel que se forma alrededor de la tableta al estar en contacto con el medio acuoso, impidiendo la difusión del agua hacia el interior de la matriz. Esto coincide con lo reportado por Mollan y Celik (1993), quienes indican que el fenómeno de gelificación superficial da lugar a tiempos de desintegración independientes de la presión de compactación, a lo que Herman y Remon (1989) agregan que la desintegración de las tabletas de almidón pregelatinizado está condicionada por el grado de hinchamiento de los granos.

Las tabletas elaboradas con almidón de malanga tienen un valor de espesor diferente que las otras, además de que hay diferencia significativa entre estos valores $(P \leq 0.05)$. Comparando los resultados con lo publicado por Bastos, Friedrich y Beck (2008), presentan rangos de espesor más elevados (4.62-4.72 $\mathrm{cm})$, argumentando que fueron similares para las diferentes formulaciones de acuerdo con la matriz y los punzones utilizados en su trabajo.

Por su parte, Monedero, Muñoz, Velasco, Muñoz y Jiménez (1996) aseguran que existe una buena correlación entre los valores del índice de compresibilidad (IC) cuando son $\leq 20 \%$ de los almidones para compresión directa y la variación de peso de las tabletas. Esto concuerda con los resultados obtenidos para el peso de las tabletas, los cuales nos dicen que no hay diferencia significativa en las tabletas con respecto al peso, aunque el peso promedio de aquellas elaboradas con almidón de malanga es ligeramente inferior comparado con el de las tabletas de los otros dos tratamientos; entonces se puede decir que este peso se vio afectado por el tamaño de partícula. Por otro lado, Peña y Pereda (1995) reportaron valores parecidos para tabletas elaboradas con PVP y almidón comparadas con comprimidos comerciales de ácido acetilsalicílico (590-635.6 mg y 623.3-631.4 mg vs 590-635.6 mg), exhibiendo diferencias estadísticamente significativas, lo que concuerda con los resultados en peso de las tabletas obtenidas en el presente estudio.

Con respecto al diámetro, se observó que sólo se presentó discrepancia significativa con las tabletas de aspirina ( $P \leq 0.05)$, en relación con las de almidón de papa y de malanga, que tienen el mismo diámetro. Comparando los resultados con los obtenidos por Bastos, Friedrich y Beck (2008), éstos fueron menores.

La compactabilidad está asociada a la dureza de las tabletas, que deben tener suficiente resistencia mecánica ante el desmoronamiento o la rotura cuando se manipulan o procesan, especialmente durante el empaque. La dureza de la tableta es, por lo tanto, importante y tiene relevancia práctica (llića, Kása, Dreua, Pintye-Hódib y Srčiča, 2010). Los resultados obtenidos muestran una diferencia significativa entre los valores de dureza $(P \leq 0.05)$ de las tabletas elaboradas con almidones de malanga y papa respecto de las comerciales. Esto concuerda con lo reportado por Bastos, Friedrich y Beck (2008). Acerca de la dureza de formulaciones, el estudio mostró una influencia considerable $(p \leq 0.05)$ para ambos factores evaluados (granulometría de aglomerante de relleno y lubricante) sin interacción entre ellos.

\section{Conclusiones}

La malanga cultivada en la región de Tuxtepec, Oaxaca, se podría considerar como materia prima de gran potencial debido a su elevado contenido de almidón, con aplicaciones en la industria alimenticia y farmacéutica. 
El almidón extraído de la malanga cumplió con los estándares en los ensayos de identidad y pruebas reológicas, lo que permite sugerir que es apto para ser utilizado como excipiente en la elaboración de tabletas o comprimidos. Las tabletas fabricadas con el almidón de malanga tienen buenas características de dureza y desintegración, por lo que los resultados alcanzados permiten considerar su posible uso como excipiente para compresión directa.

Se plantea la necesidad de realizar estudios que permitan determinar la factibilidad de la obtención del almidón de malanga y profundizar en el análisis de las propiedades farmacéuticas del mismo.

\section{Referencias}

Agbor-Egbe, T. y Rickard, J. E. (1991). Study on the factors affecting storage of edible aroids. Annals of Applied Biology, 119, 121-130. Disponible en https://onlinelibrary.wiley.com/doi/ pdf/10.1111/j.1744-7348.1991.tb04850.x

Ansel, H. C. y Popovich, N. G. (1993). Pharmaceutical Dosage Forms and Drug Delivery Systems. Philadelphia, PA: Lea and Febiger.

Arancibia, A., Chávez, J., Costa, E., Nella, G. M. y Thielemann, A. M. (1994). Tecnología farmacéutica. Chile: Departamento de Ciencias y Tecnología Farmacéutica, Universidad de Chile.

Bastos, M. O., Friedrich, R. B. y Beck, C. R. (2008). Effects of filler-binders and lubricants on physicochemical properties of tablets obtained by direct compression: A 22 factorial design. Lat. Am. J. Pharm., 27(4), 578-83. Disponible en http://citeseerx.ist.psu.edu/viewdoc/ summary?doi=10.1.1.403.4197

Braverman, J. B. S. (1992). Introducción a la Bioquímica de los alimentos. México: El Manual Moderno.

Carteasen, J. T. (1990). Solid Pharmaceutical: Mechanical Properties and Rate Phenomena. New York: Academic Press.

Connors, K. A., Amidon, G. L. y Stella, V.J. (1986). Stability of pharmaceuticals. A handbook for pharmacists. New York: John Wiley.
Dendy, D. A. (2001). Composite and alternative flours. En D. A. Dendy y B. J. Dobraszczyk (eds.), Cereal Products (pp. 263-275). Aspen Publisher Inc.

Dreher, M. L., Dreher, C. J. y Berry, J. W. (1984). Starch digestibility of foods: a nutritional perspective. Crit Rev Food Sci Nutr., 20(1), 47-71. Disponible en https:// www.ncbi.nlm.nih.gov/pubmed/6380959

Falade, K. O. y Okafor, C. A. (2013). Physicochemical properties of five cocoyam (Colocasia esculenta and Xanthosoma sagittifolium)starches. Food Hydrocolloids, 30, 173-181. Disponible en https://www.researchgate. net/publication/257102734_Physicochemical_ properties_of_five_Cocoyam_Colocasia_esculenta_ and_Xanthosoma_sagittifolium_starch

Food and Agriculture Organization [FAO]. (2014). Datos sobre alimentación y agricultura. Disponible en http://www.fao.org/faostat

FAO y WHO. (2002). Diet, nutrition and the prevention of chronic diseases. Report of a joint FAO/WHO expert consultation, vol. 916. Ginebra: autores.

Garzón S., M. L. (2006). Almidón retrogradado para su uso en compresión directa. II. Compactación de almidón de chayote retrogradado. Revista Mexicana de Ciencias Farmacéuticas, 37(3), 29-39. Disponible en https:// www.redalyc.org/pdf/579/57937305.pdf

Gujska, E., D-Reinhard, W. y Khann, K. (1994). Physicochemical properties of field pea, pinto and nay bean starches. J. Foods Sci., 59(3), 634-637. Disponible en https://onlinelibrary.wiley.com/doi/ abs/10.1111/j.1365-2621.1994.tb05580.x

Herman, J. y Remon, J. P. (1989). Modified starches as hidrophilic matrices for controlled oral delivery. II In vitro drug release evaluation of thermally modified starches. International Journal of Pharmaceutics, 56, 65-70. Disponible en https://www.sciencedirect.com/ science/article/pii/0378517389900616

Holleman, L. W. J. y Aten, A. (1956). Processing of cassava and its products. Roma: FAO.

Ilića, I., Kása, P., Dreua, R., Pintye-Hódib, K. y Srčiča, S. (2010). A modification of the Pr value equation for measuring the compactibility of pharmaceutical materials. Chemical Engineering and Processing: 
Process Intensification, 49(8), 881-884. Disponible en https://www.sciencedirect.com/science/article/pii/ S0255270110001340

Instituto Nacional de Nutrición. (1999). Tablas de Composición de Alimentos. México: Instituto Nacional de Nutrición "Salvador Zubirán".

Kaur, M., Kaushal, P. y Sandhu, K. S. (2013). Studies on physicochemical and pasting properties of Taro (Colocasia esculenta L.) flour in comparison with a cereal, tuber and legume flour. J. Food Sci Technol,, 50(1), 94-100. Disponible en https://www.ncbi.nlm. nih.gov/pubmed/24425892

Kaushal, P., Kumar, V. y Sharma, H. K. (2012). Comparative study of physicochemical, functional, antinutritional and pasting properties of taro (Colocasia esculenta), rice (Oryza sativa) flour, pigeonpea (Cajanus cajan) flour and their blends. LWT - Food Science and Technology, 48, 59-68. doi 10.1016/j. Iwt.2012.02.028

Langeland, K. A. y Burks, K. C. (1998). Identification and biology of non-native plants in Florida's natural areas. USA: University of Florida.

Linoya, K., Gotoh, K. y Higashitani, K. (1995). Powder Technology Handbook. New York: Marcel Dekker.

Mollan, M. J. y Celik, M. (1993). Characterization of directly compressible maltodextrins manufactured by three different processes. Drug Development and Industrial Pharmacy, 19(17\&18), 2335-2358. Disponible en https://www.tandfonline.com/doi/ abs/10.3109/03639049309047194

Monedero, P. M., Muñoz, R. A., Velasco, A. M., Muñoz, M. N. y Jiménez C., R. M. (1996). Comparative tableting microstructural properties of a new starch for direct compression. Drug Development and Industrial Pharmacy, 22(7), 689-695. doi 10.3109/03639049609063225

Musumara, G., Scarlala, G. y Cirma, G. (1985). Identification of drugs by principal components analysis of thin layer chromatography in four eluent systems. J. Chromatgr., 350, 151-68. Disponible en https://www.ncbi.nlm.nih.gov/pubmed/4093478

Mweta, D. E., Labuschagne, M. T., Koen, E., Benesi, I. R. M. y Saka, J. D. K. (2008). Some properties of starches from cocoyam (Colocasia esculenta) and cassava (Manihot esculenta Crantz.) grown in Malawi. African Journal of Food Science, (2), 102-111. Disponible en https://academicjournals.org/journal/AJFS/article-fulltext-pdf/5AA6F1417075

Nwokocha, L. M., Aviara, N. A., Senan, C. y Williams, P. A. (2009). A comparative study of some properties of cassava (Manihot esculenta, Crantz) and cocoyam (Colocasia esculenta, Linn) starches. Carbohydrate Polymers, 76, 362-367. Disponible en https:// www.sciencedirect.com/science/article/pii/ S0144861708004980?via\%3Dihub

Onwueme, I. (1999). Taro cultivation in Asia and the Pacific. En Food and Agriculture. Bangkok: Organization (FAO) of the United Nations Regional office for Asia and the Pacific.

Parrot, E. L. (1971). Pharmaceutical Technology: Fundamental Pharmaceutics. Minneapolis: Burgess Publishing Company, $3^{\text {a }}$. ed.

Peña, L. A. y Pereda, R. E. (1995). Efecto de la polivinilpirrolidona, el almidón y el laurilsulfato de sodio sobre la disolución de comprimidos de ácido acetilsalicílico. Bioequivalencia in vitro. Acta Farm. Bonaerense, 14(2), 107-118. Disponible en http:// sedici.unlp.edu.ar/handle/10915/7070

Pérez, E., Schultz, F. S. y Pacheco, D. E. (2005). Characterization of some properties of starches isolated from Xanthosoma sagittifolium (tannia) and Colocassia esculenta (taro). Carbohydrate Polymers, 60, 139-145. Disponible en http:// www.academia.edu/6086297/Characterization_ of_some_properties_of_starches_isolated_from_ Xanthosoma_sagittifolium_tannia_and_Colocassia_ esculenta_taro

Peroni, F., Rocha, T. y Franco, C. (2006). Some structural and physicochemical characteristics of tuber and root starches. Food Sci. Tech. Int., 12(6), 505-513. Disponible en https://journals.sagepub.com/ doi/10.1177/1082013206073045

Riley, C. K., Wheatley, A. O. y Asemota, H. N. (2006). Isolation and characterization of starches from eight Dioscorea alata cultivars grown in Jamaica. Afri. J. Biotechnol., 5(17), 1528-1536. Disponible en https://www.ajol.info/index.php/ajb/article/ viewFile/43151/26694 
Rincón, A., Araujo, V. C., Padilla, F. y Martín, E. (2000). Evaluación del posible uso tecnológico de algunos tubérculos de las dioscóreas: ñame congo (Dioscorea bulbífera) y mapuey (Discorea trífida). Arch. Latinoamer. Nutr., 50(3), 286-290. Disponible en http://www.scielo.org.ve/scielo.php?script=sci_ arttext\&pid=S0004-06222000000300012

Rodríguez-Miranda, J., Rivadeneyra-Rodríguez, J. M., Ramírez R., E. J., Juárez B., J. M., Herrera, T. E., Navarro C., R. 0. y Hernández, S. B. (2011). Caracterización fisicoquímica, funcional y contenido fenólico de harina de malanga (Colocasia esculenta) cultivada en la región de Tuxtepec, Oaxaca, México. Ciencia y Mar. XV(43), 37-47. Disponible en http://www.umar.mx/ revistas/43/0430104.pdf

Rodríguez-Miranda, J., Ruiz-López, I. I., Herman-Lara, E., Martínez-Sánchez, C. E., Delgado-Licon, E. y Vivar-Vera, M. A. (2011). Development of extruded snacks using taro (Colocasia esculenta) and nixtamalized maize (Zea mays) flour blends. LWT - Food Science and Technology, 44, 673-680. Disponible en https://www.infona.pl/ resource/bwmeta1.element.elsevier-7359c1f2-e4bc3d11-9bd1-b25c90ab32d9

Secretaría de Salud. (2000). Farmacopea de los Estados Unidos Mexicanos, tomos I y II. México: autor.
Srichuwong, S., Sunarti, T. C., Mishima, T., Isono, N. y Hisamatsu, M. (2005). Starches from different botanical sources II: Contribution of starch structure to swelling and pasting properties. Carbohydrate Polymers, 62(1), 25-34. Disponible en https://www.infona.pl/resource/ bwmeta1.element.elsevier-4e5e4564-e894-3ac18346 -aedc50875c7f

Tattiyakul, J., Asavasaksakul, S. y Pradipasena, P. (2006). Chemical and physical properties of flour extracted from Taro Colocasia esculenta (L.) schott grown in different regions of Thailand. Science Asia, 32, 279-284. Disponible en http://citeseerx.ist.psu.edu/viewdoc/ download?doi=10.1.1.611.7620\&rep=rep1\&type=pdf

Tester, R. F., Yusuph, M., Millam, S. y Davies, H. V. (2004). Effects of temperature on the physicochemical properties of starches extracted from microtubers grown from different potato cultivars. J. Sci. Food Agric., 84(11), 1397-1404.

Tjahjadi, C., Lin, S. W. y Breene, W. M. (1988). Isolation and characterization of adzuki bean (Vigna angularis cv Takara) proteins. Journal of Food Science, 53, 14381443. Disponible en https://onlinelibrary.wiley.com/ doi/abs/10.1111/j.1365-2621.1988.tb09294.x

Vila Jato, J. L. (1997). Tecnología farmacéutica, volumen II. Madrid: editorial Síntesis. 


\section{Cuadro 1.}

Pruebas reológicas

\begin{tabular}{|l|c|c|}
\hline & ALMIDÓN DE PAPA & ALMIDÓN DE MALANGA \\
\hline Humedad $(\%)$ & 10.82 & 11.00 \\
\hline Ph & 6.5 & 6.6 \\
\hline Tamaño de partícula $(\mu \mathrm{m})$ & 248.9 & 248.9 \\
\hline Ángulo de reposo $\left({ }^{\circ}\right)$ & 35.0 & 48.0 \\
\hline Velocidad de flujo $(\mathrm{g} / \mathrm{cm} 2 . \mathrm{s})$ & 8.98 & 7.40 \\
\hline Densidad aparente $(\mathrm{g} / \mathrm{mL})$ & 0.78 & 0.72 \\
\hline Densidad compactada $(\mathrm{g} / \mathrm{mL})$ & 0.89 & 0.92 \\
\hline Índice de Hausner & 0.88 & 0.78 \\
\hline
\end{tabular}

\section{Cuadro 2.}

Análisis de tabletas

\begin{tabular}{|c|c|c|c|}
\hline & ASPIRINA & ALMIDÓN DE PAPA & ALMIDÓN DE MALANGA \\
\hline \multicolumn{4}{|c|}{ Tiempo de desintegración (seg) } \\
\hline Media $\pm D E$ & $11.5 \pm 1.5 \mathrm{~b}$ & $19.7 \pm 0.7 \mathrm{c}$ & $4.3 \pm 0.1 \mathrm{a}$ \\
\hline Amplitud & $10.0-13.0$ & $19.0-20.5$ & $4.1-4.4$ \\
\hline \multicolumn{4}{|l|}{ Espesor $(\mathrm{cm})$} \\
\hline Media $\pm \mathrm{DE}$ & $0.53 \pm 0.06 a$ & $0.51 \pm 0.02 \mathrm{a}$ & $0.57 \pm 0.03 \mathrm{~b}$ \\
\hline Amplitud & $0.39-0.57$ & $0.47-0.55$ & $0.51-0.63$ \\
\hline \multicolumn{4}{|l|}{ Diámetro $(\mathrm{cm})$} \\
\hline Media $\pm \mathrm{DE}$ & $1.16 \pm 0.07 \mathrm{~b}$ & $1.0 \pm 0.0 \mathrm{a}$ & $1.0 \pm 0.0 \mathrm{a}$ \\
\hline Amplitud & $1.0-1.2$ & $1.0-1.0$ & $1.0-1.0$ \\
\hline \multicolumn{4}{|l|}{ Dureza (Kg) } \\
\hline Media \pm DE & $11.67 \pm 0.41 \mathrm{a}$ & $12.40 \pm 0.5 \mathrm{~b}$ & $12.97 \pm 0.41 c$ \\
\hline Amplitud & $11.0-12.5$ & $11.5-13.0$ & $12.5-13.5$ \\
\hline \multicolumn{4}{|l|}{ Peso (g) } \\
\hline Media $\pm D E$ & $0.59 \pm 0.003 c$ & $0.59 \pm 0.006 b$ & $0.58 \pm 0.005 \mathrm{a}$ \\
\hline Amplitud & $0.59-0.60$ & $0.58-0.60$ & $0.57-0.60$ \\
\hline
\end{tabular}

\section{Cuadro 3.}

Ángulo de reposo

\begin{tabular}{|l|c|}
\hline \multicolumn{1}{|c|}{ DESEMPEÑO DE LOS ALMIDONES } & ÁNGULO DE REPOSO \\
\hline Muy móviles & $25^{\circ}$ a $30^{\circ}$ \\
\hline Móviles & $30^{\circ}$ a $38^{\circ}$ \\
\hline Aceptable movilidad & $38^{\circ}$ a $45^{\circ}$ \\
\hline Cohesivos (poca movilidad) & $45^{\circ}$ a $55^{\circ}$ \\
\hline Muy cohesivos (muy mala movilidad) & $55^{\circ}$ hasta $70^{\circ}$ \\
\hline
\end{tabular}

